\title{
Male reproduction contribution down to two genes
}

The Y chromosome encodes genes important for natural male reproduction, but when it comes to assisted reproductive technologies, not all of those genes may be necessary to produce viable offspring. In fact, a new study shows that only two Y chromosome genes are needed to generate live mouse offspring with assisted reproduction: Sry, a gene critical for the development of testes, and Eif2s $3 y$, which is needed to initiate spermatogenesis (Science published online 21 November 2013; doi:10.1126/science.1242544).

Monika Ward and her colleagues at University of Hawaii, Honolulu, previously demonstrated that, with the use of assisted reproduction, live offspring could be obtained from mice lacking the entire Y chromosome long arm; the remaining Y chromosome in these mice only encoded seven genes and three gene families. But now the team has further narrowed down the essential Y contribution to two genes, Sry and Eif2s3y.

The transgenic male mice with only these two Y genes were considered infertile because the germ cells that normally develop into sperm did not fully mature in these mice, instead remaining as round spermatids. The team harvested these immature, often abnormally shaped spermatids and used a technique called round spermatid injection (ROSI) to inject them into healthy female mouse eggs. When the successfully fertilized oocytes were transplanted into the oviducts of recipient females, live offspring were obtained. Furthermore, these offspring were capable of breeding.

"When it comes to assisted reproduction," the authors wrote, "our mouse study proves that the Y chromosome contribution can be brought to a bare minimum consisting of Sry and Eif2s3y. Indeed, it may well be possible to eliminate the mouse Y chromosome altogether if appropriate replacements are made for those two genes."

That is not to say that the remaining genes on the Y chromosome do not serve important functions in male fertility. The overall efficiency of ROSI with two Y genes was only $9 \%$, much lower than the $26 \%$ success

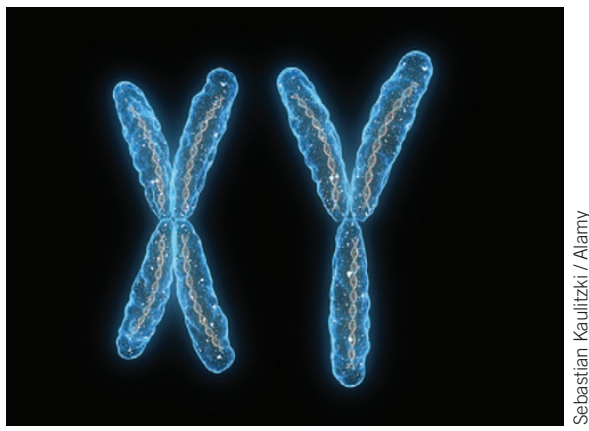

rate of ROSI with spermatids from normal $\mathrm{XY}$ males. When the researchers replaced Sry with the sex reversal factor Sxrb, which encodes three additional Y genes, as well as a partial chromosome to pair with the partnerless X chromosome during cell division, they were able to achieve ROSI success rates as high as $20 \%$. As the authors explained, "Human Y chromosome...genetic information is undoubtedly important for many aspects of reproduction involving the development of mature sperm and its function in normal fertilization."

Kara Rosania

\section{COPPER PROMOTES TUMOR GROWTH}

New study results from researchers at the Swiss Federal Institute of Technology Lausanne (Switzerland) suggest that copper is essential for energy production in malignant cells and that reducing levels of bioavailable copper can slow tumor growth in mice.

The trace element copper is key to cell growth because of its role in energy production through respiration. Cell growth requires energy, which is stored molecularly as adenosine triphosphate (ATP) after being produced by either respiration or glycolysis. Respiration is the more efficient of the two processes but involves several enzymes, one of the most important of which requires copper for its activity. Its role in cellular energy production may help to explain why copper is important in cancer. As cancer cells typically undergo rapid proliferation, their energy requirements are greater than those of normal cells. Indeed, copper imbalances have previously been associated with cancer as well as other diseases.

Seiko Ishida, who led the new study along with Douglas Hanahan, explained in a press release, "This study was motivated by our previous puzzling observation...that cancers, unlike healthy tissues, are especially sensitive to changes in systemic copper levels."

To investigate the association between copper and cancer, the researchers used genetically engineered mice that develop pancreatic neuroendocrine tumors. They exposed some of the mice to copper by spiking their drinking water with copper at the maximum levels permitted in public water supplies by the US Environmental Protection Agency $(20 \mu \mathrm{M})$. In a second group of mice, they reduced systemic copper levels using the copper-chelating drug tetrathiomolybdate. Copper exposure accelerated tumor growth in mice, whereas copper insufficiency reduced tumor growth (Proc. Natl. Acad. Sci. USA published online 11 November 2013; doi:10.1073/ pnas.1318431110). Copper-deficient tumors seemed to be compensating for a lack of copper by using glycolysis rather than respiration to produce energy but were unable to produce sufficient ATP and did not grow.

The results provide evidence that copper can enhance the proliferation of cancer cells, "indicating that copper is an essential nutrient for them," according to Ishida.

If the same holds true for tumors in humans, then controlling copper levels in cancer patients could be clinically beneficial. Ishida and Hanahan's team proposes that minimizing systemic copper levels, combined with blocking glycolysis, could offer a two-step strategy to 'starve' cancer cells by limiting the two major pathways for ATP production. Animal studies should be developed to evaluate the therapeutic potential for such a strategy. 